modèles $+\cdots \cdot \ldots+$ !

linguistiques "थं:

\section{Modèles linguistiques}

$53 \mid 2006$

La préposition en français (I). Philologie et linguistique diachronique (domaine anglais)

\title{
3. Sur le statut des locutions prépositives
}

\section{Gaston Gross}

\section{(2) OpenEdition}

\section{Journals}

Édition électronique

URL : https://journals.openedition.org/ml/517

DOI : $10.4000 / \mathrm{ml} .517$

ISSN : 2274-0511

Éditeur

Association Modèles linguistiques

\section{Édition imprimée}

Date de publication : 1 janvier 2006

Pagination : 35-50

\section{Référence électronique}

Gaston Gross, «3. Sur le statut des locutions prépositives », Modèles linguistiques [En ligne], 53 | 2006, mis en ligne le 01 février 2015, consulté le 21 septembre 2021. URL : http://journals.openedition.org/ ml/517 ; DOl : https://doi.org/10.4000/ml.517

Ce document a été généré automatiquement le 21 septembre 2021.

(c) Modèles Linguistiques 


\title{
3. Sur le statut des locutions prépositives
}

\author{
Gaston Gross
}

\section{Un problème de terminologie}

1 Les grammaires nous enseignent que parallèlement aux catégories grammaticales simples, il en existe de composées, dont la dénomination cependant n'est pas constante. On parle de locutions verbales, adjectivales, prépositionnelles, conjonctives, adverbiales mais, en revanche, de noms composés. Les déterminants complexes n'ont pas droit, eux, à un statut particulier. Souvent aussi on confond la composition, procédé de formation d'unités polylexicales, avec le figement, qui désigne l'opacité sémantique de la séquence. Ainsi, sous le terme de «locutions ", on range des suites très hétérogènes, comme on le verra dans la suite de cet article.

D'autres objections méritent d'être formulées à propos de la terminologie grammaticale. Par exemple, on distingue les locutions "prépositives» des locutions " conjonctives », comme s'il s'agissait de catégories différentes. Or, ce qui sépare afin de de afin que, ce n'est pas le statut du connecteur lui-même, mais la nature du complément : la forme en que $P$ introduit un prédicat conjugué et celle en de le même prédicat à l'infinitif, c'est-à-dire dépourvu d'actualisation. Il n'y a donc aucune raison d'attribuer un nom différent à ces deux variantes mineures de la «locution». Cette différence s'explique, en outre, de façon simple. En cas de coréférence des sujets de la principale et de la subordonnée, le prédicat de cette dernière perd son actualisation et hérite de celle du verbe principal. On voit donc que les phénomènes en cause sont extérieurs à la locution et n'ont aucune incidence sur son statut. Il n'y a aucune raison de voir dans ce phénomène une différence de catégorie.

Une autre observation concerne l'opposition entre la notion de complément et celle de subordonnée circonstancielle. La distinction classique semble claire : on a affaire à un complément quand la préposition introduit un nom et à une subordonnée quand il s'agit d'une phrase. Ainsi sans tablier est un complément et sans qu'il puisse dire un mot 
est une proposition subordonnée. C'est oublier qu'il existe des substantifs prédicatifs. Ces derniers, étant des prédicats, constituent le noyau d'une phrase autonome. Si sans tablier est un complément, ce n'est pas le cas de sans peur qui est une variante de sans avoir peur, réduction infinitive de sans qu'il ait peur. On doit donc considérer toute préposition ou locution prépositive suivie d'un substantif prédicatif comme une proposition subordonnée. Ces considérations facilitent grandement les analyses que nous faisons dans la suite de cet article.

\section{Formations morphologiques}

Nous ne nous intéressons pas ici à la formation des prépositions simples. Leur origine est signalée par la plupart des grammaires universitaires. En revanche, nous soulignons les différentes formes morphologiques que peuvent prendre les locutions prépositives (ou conjonctives).

a. Groupes nominaux avec préposition : à force de, par rapport à, au lieu de, à l'occasion de, à l'aide de, en raison de. Certains de ces groupes peuvent comprendre un adjectif intensif : au fin fond de, en plein milieu de, en plein coeur de, au beau milieu de, au plus fort de (la mêlée), au grand dam de. Dans d'autres groupes, on observe une répétition du substantif : au coude à coude avec, en tête à tête avec.

b. Groupes nominaux sans préposition introductrice : faute de, suite à, côte à côte avec.

c. Infinitifs introduits par une préposition : à dater de.

d. Formations adverbiales suivies d'une préposition : conformément à, loin de.

e. Ablatifs absolus suivis d'une préposition : abstraction faite de, compte tenu de.

f. Ablatifs absolus avec effacement du participe : à part les premiers, mis à part les premiers.

Ces structures peuvent subir certaines modifications. Tout d'abord, il existe des transitions entre certaines formes. Ainsi, un ablatif absolu peut être mis en parallèle avec une forme nominale: Tous sont venus, excepté Jean; Tous sont venus à l'exception de Jean. Les locutions peuvent être soudées dans certaines conditions, comme dans afin que. Cette soudure est cependant superficielle, car en présence d'un article, elle disparaît : à telle fin que. Une même racine est susceptible d'avoir plusieurs réalisations morphologiques: on trouve ainsi une forme nominale parallèlement à une forme adverbiale : au contraire de, contrairement $\grave{a}$; au travers de $N$, à travers $N$. Certaines locutions font l'objet de sous-structures par effacement d'un de leurs éléments constitutifs : au nez de < au nez et à la barbe de, outre que < outre le fait que, par pluie < par temps de pluie. Quelquefois, la locution comprend deux substantifs formant une structure binaire au fur et à mesure de, au nez et à la barbe de, au vu et au su de, aux risques et périls de, en lieu et place de. Enfin, il semble que certaines locutions soient issues de constructions verbales avec effacement de ce verbe. Du fait de est issu de venir du fait de, à bonne distance de exige un verbe de localisation être, se tenir, se trouver. Voici encore quelques exemples : (être) à dos de, (être) à l'origine de, (se trouver) au chevet de, (tenir) au fait que, (figurer) au nombre de, (confier qq chose) aux bons soins de, (avoir lieu) du vivant de.

\section{Origine française ou latine ?}

6 Il est dit souvent que les locutions prépositives sont de création française, les prépositions issues du latin sont simples. Ce point de vue n'est pas entièrement exact. Il 
existait en latin des locutions prépositives formées à l'aide d'un substantif. Je dois les informations qui suivent à Michèle Fruyt et à Huguette Fugier, que je remercie vivement. Plusieurs substantifs latins se sont prêtés à la formation de ces constructions. Et tout d'abord causa, dans son sens causal, a donné : génitif + causa (à l'ablatif) : à cause $d e$, en vue de, honoris causa : pour honorer, par honneur ; vestra reique publicae causa (Cic.) - « dans votre intérêt comme dans l'intérêt général ». Cette construction est assez libre du point de vue de la détermination : qua causa? «pour quelle raison?»; ea causa: " pour cette raison ». Il peut y avoir adjonction d'une autre préposition : eadem de causa, isdem de causis; qua de causa, quibus de causis; qua ex causa ; ob eam causam, ob eas causas, ob eam ipsam causam quod (Cicéron), ob eam unam causam quia (Cicéron), ob eamdem causam; ea causa ut = idcirco ut, ideo ut : " pour que, afin que ».

7 Un autre substantif est gratia, qui signifie reconnaissance. A l'ablatif et précédé d'un génitif, il signifie à cause de, pour l'amour de, pour - hominum gratia: "pour les hommes"; honoris gratia: "pour l'honneur», exempli gratia: "pour prendre un exemple» (Cic.); mea gratia : «à cause de moi » (Plaute). Ce substantif permet aussi de former une conjonction de subordination causale - ea gratia quod: "pour ce motif que ». Beneficium (bienfait) permet la formation de génitif + beneficio : "grâce à ». Le substantif au génitif uicis, inusité au nominatif, tour, alternative (le tour de quelqu'un, sa place, son rôle) permet avec un figement à l'ablatif (uice) une locution signifiant à la place de, en guise de, comme: salis uice (Pline) : « en guise de sel»; alicuius uice: «à la place de quelqu'un ».

Le substantif species au sens de vue, regard, apparence permet de former sub specie tutelae: «sous prétexte de tutelle» (Quinte-Curce); per speciem auxilii ferendi: «sous couleur de porter secours». Le substantif res (chose) permet de former plusieurs locutions: quamobrem: conjonction de subordination causale - multae sunt causae quamobrem cupiam (Térence): «il y a beaucoup de raisons, pour lesquelles je désirerais "; ou encore quare: conj. de subordination causale - en raison de quoi et adverbe interrogatif pourquoi? Enfin le substantif modus (manière) forme à son tour eomodo - « de cette manière "; eodem modo : « de la même manière ", et le subordonnant quomodo. On peut donc dire que les procédés de formation des locutions, qui font l'objet de cet article, étaient déjà en place en latin classique.

\section{Les locutions prépositives sont-elles figées?}

\subsection{Le substantif}

9 La notion même de locution implique, de façon plus ou moins explicite, une restriction des propriétés combinatoires. Il se trouve seulement que le figement n'est pas une propriété binaire. Il existe des degrés de figement (G. Gross 1988) et il peut affecter tout ou partie de la séquence. Les restrictions, dans le cas d'une locution, concernent soit le substantif lui-même, soit la préposition, soit la détermination. On dira que le substantif n'est pas figé quand il a le même sens et, de façon générale, le même emploi que dans une position prédicative. Ainsi le substantif intention dans avec l'intention de n'est figé ni sémantiquement ni syntaxiquement, car il a les mêmes propriétés que dans avoir l'intention de. Cette liberté s'observe dans la plupart des locutions prépositives ou conjonctives. S'il est figé, le substantif a un fonctionnement différent de celui des expressions libres: le substantif dépit dans en dépit de ne peut pas être interprété 
comme un nom de sentiment; coup dans à coups de (subventions) n'a rien à voir avec l'idée de battre; dans à l'article de (la mort) le substantif ne désigne pas une partie du discours. Voici encore quelques exemples : au grand dam de, au seuil de (la nouvelle année), aux bons soins de, au nez et à la barbe de, au lieu de, en guise de, en vertu de. Nous verrons plus loin que, dans les locutions " libres ", la plupart des substantifs sont des prédicats nominaux non actualisés, qui peuvent recevoir une actualisation autonome à l'aide de verbes supports, comme c'est le cas de intention ci-dessus.

\subsection{La préposition}

La plupart du temps, la préposition qui introduit la locution est unique mais l'existence de paradigmes en cette position n'est pas un fait exceptionnel. Avec le substantif aide, on peut avoir à ou avec : à l'aide de / avec l'aide de. Le substantif but accepte avec et dans : avec pour but de / dans le but de, de même que le substantif intention. De et en sont possibles avec sorte : de sorte que / en sorte que. Crainte accepte par et de : par crainte de / de crainte de. Il existe une alternance par / zéro avec crainte : par crainte de VW / crainte de $V W$. Ces alternances de prépositions sont fréquentes avec un substantif locatif (au bas de, en bas de, dans le bas de ; à bord de, au bord de, sur le bord de) ou temporel (à la fin de, sur la fin de, vers la fin de).

\subsection{La détermination}

11 Les variations sont encore plus significatives dans le cas de la détermination. C'est une des raisons pour lesquelles on peut affirmer que la plupart des locutions ne sont pas figées mais constituent des configurations syntaxiques particulières de constructions prédicatives. En effet, on observe des déterminants :

- négatifs : dans le but précis de $\mathrm{VW} /$ dans aucun but précis ; pour le motif que / pour nul autre motif; au moment où / à aucun moment ;

- interrogatifs : pour quelle raison? pour quel motif? à quel moment? dans quelles conditions? dans quel but?

- exclamatifs : et dans quelles conditions ! et à quelle fin ! et à quel moment !

- indéfinis : pour d'autres raisons, à un certain moment, dans d'autres circonstances, pour n'importe quelle raison, pour telle ou telle raison;

- quantifieurs : pour deux raisons, pour plusieurs raisons : la première... la seconde.., pour les deux motifs suivants.

On remarquera que cette liberté de la détermination exclut que l'on puisse conserver sans modifications la dénomination de locutions prépositives. Cette constatation est encore plus justifiée si on prend en compte les phénomènes d'anaphore. Les déterminants dans les "locutions» ont un emploi cataphorique: ils annoncent le complément ou la subordonnée circonstancielle. Dans avec l'intention de plaire, l'article le annonce la subordonnée. Ce rôle est bien connu et c'est le seul qui soit envisagé dans le cadre des locutions. Mais un autre cas de figure n'est jamais évoqué. C'est celui où le contenu sémantique d'un complément ou d'une circonstancielle correspond à un fait déjà connu de l'interlocuteur. Dans ce cas, on ne répète pas l'information mais on y renvoie à l'aide d'éléments anaphoriques. Voici l'essentiel de ces déterminants. 


\subsubsection{L'anaphore se réduit au modifieur} d'une pronominalisation dont le but est de référer à un fait antérieur connu. $\mathrm{Ce}$ pronom a différentes formes :

- le relatif de liaison :

en conséquence de ce que $P$ / en conséquence de quoi

au lieu de ce que $P$ / au lieu de quoi

faute de ce que P / faute de quoi

après que $P$ / après quoi

- les pronoms cela, ça et là :

en comparaison de ce que $\mathrm{P} /$ en comparaison de $($ cela $+c ̧ a)$

en conséquence de ce que $\mathrm{P} /$ en conséquence de $($ cela $+c ̧ a)$

après que $P$ / après (cela + ça)

à cause de ce que $P$ / à cause de cela

d'ici à ce que $P$ / d'ici là

A faut $(E+$ de pouvoir lire), je me promènerai

A force ( $E+$ de crier comme ça), tu te fatigueras

\subsubsection{L'anaphore affecte toute la détermination : le démonstratif}

Le déterminant cataphorique leMc (où $M c$ désigne la subordonnée circonstancielle, qui est en fait une complétive, G. Gross 2005) peut être remplacé par le démonstratif ce, qui renvoie à un événement antérieur considéré comme connu. Il s'agit du cas d'anaphore le plus fréquent. En voici quelques exemples : 


\subsection{Insertion d'adjectifs} substantif figurant dans la locution; ainsi le démonstratif ce dans dans ce but correspond à l'ensemble constitué par l'article cataphorique le et la subordonnée finale considérée comme un modifieur: dans le but de plaire, dans ce but. Ce type d'anaphore s'observe aussi en l'absence d'un article défini: afin de plaire, à cette fin. Nous appellerons ce cas de figure une anaphore interne, puisqu'il s'agit de la détermination du substantif prédicatif figurant dans la locution. Cette anaphore peut aussi être prise en charge par un possessif : à l'initiative de Paul, à son initiative; dans l'intérêt de Jean, dans son intérêt ; à l'avantage de Luc, à son avantage. cette fin, à cet effet, dans ces conditions où l'anaphore correspond à la détermination du substantif, on observe des cas d'impossibilité de cette nature : en vue de plaire, ${ }^{*}$ en cette vue; en raison d'un épais brouillard, *en cette raison; à cause de l'absence de Paul, *à cette cause. Ici, l'anaphore porte sur le complément exclusivement : en vue de cela, en raison de cela, à cause de cela. Nous appellerons cette configuration anaphore externe. la détermination du substantif but change : une détermination cataphorique le-modif où le modifieur est constitué par la subordonnée ou, à l'occasion, par un complément est remplacée par une détermination anaphorique où ce est l'équivalent de le-modif. En revanche, dans à cause de l'anaphore n'affecte que le complément.

L'adjonction d'adjectifs dans une structure est un argument supplémentaire pour souligner la liberté syntaxique des locutions. Cette situation est très fréquente. On ne donnera que quelques exemples pour illustrer le phénomène. On prendra les mots but et raison:

Dans le but de: dans le but évident de, dans un but louable, dans un tel but, dans un but de ce genre que, pour des raisons similaires, pour des raisons de ce genre

la centaine. On conclura que, du fait de cette grande liberté syntaxique, la notion de locution, qui implique, qu'on le veuille ou non, un certain degré de figement, est une très mauvaise dénomination.

\subsection{Anaphore interne et externe}

Observons que, dans ce cas, l'absence d'article est de rigueur : *à une cause de, * ${ }^{*}$ une vue de, *à la cause de, *en la vue de. Il ne s'agit donc pas d'un article zéro facultatif comme dans sous prétexte de, sous le prétexte de. Examinons d'abord à cause de. Le substantif n'est pas figé puisqu'il constitue le même emploi que dans A est la cause de B. Dans cette construction, si on veut thématiser l'objet, différentes possibilités s'offrent à nous : l'opérateur à lien $B$ a A comme cause, ou encore la construction converse de nature consécutive $B$ est la conséquence de A, ou encore le passif nominal B est à cause de A. Il existe des prépositions qui permettent de former des passifs nominaux (G. Gross 1993). La forme passive à cause de ne permet qu'une anaphore externe puisqu'elle n'a pas de 
liberté syntaxique interne. La même opération peut être faite avec la racine abri $(t)$. Un toit de métal abrite cette machine donne au passif Cette machine est abritée (par, sous) un toit de métal. Parallèlement, on a une construction nominale : Un toit de métal (est, constitue, forme) un abri pour cette machine. Le passif est introduit là aussi par la préposition à : Cette machine est à l'abri d'un toit de métal. Ici il n'existe de même qu'une anaphore externe : La machine est à l'abri de ce toit, *La machine est à cet abri.

\subsection{Variation morphologique du substantif de la locution}

la préposition initiale : à l'exception de, à l'instar de, à la faveur de, aux yeux de, dans la mesure de, de mémoire de. Ailleurs, on peut trouver une motivation syntaxique. Dans un grand nombre de cas, la préposition avec peut être considérée comme une variante non actualisée du verbe avoir ou porter: Paul est venu, avec un chapeau; Paul est venu, il (avait, portait) un chapeau. Cette observation vaut si avoir est un verbe support: Paul est venu avec l'intention de gagner; Paul est venu, il avait l'intention de gagner. Le problème que nous soulevons est important mais n'a guère été traité. Il y a là à coup sûr un sujet de thèse !

\section{Les fonctions des prépositions simples}

vant d'étudier les différentes fonctions des locutions prépositives, nous allons examiner le rôle syntaxique joué par les prépositions simples. Nous allons étudier contrastivement le comportement des « locutions ». Tout d'abord, c'est bien connu, les prépositions ont comme fonction principale d'introduire les arguments des prédicats à construction indirecte : parler de $N \grave{a} N$, s'occuper de $N$, s'intéresser à $N$. Cette fonction ne mérite pas ici de plus amples développements. Elle s'applique aux prédicats nominaux comme aux adjectivaux, qui n'ont pas de constructions directes, à l'exception de l'adjectif censé. A quoi il faut ajouter que la fonction d'indicateurs d'arguments ne se résume pas à l'introduction de compléments d'objets indirects. Dans le cas du passif, la préposition 
par introduit un sujet et non pas un complément, malgré la terminologie de complément d'agent. De plus, dans les constructions nominales, la préposition de peut introduire soit un sujet (génitif subjectif: le départ de Paul) soit un objet (génitif objectif : la réquisition des troupes).

Il existe ensuite des prépositions qui ont une fonction prédicative. Partons d'abord du constat que, dans un texte, il y a autant de phrases qu'il y a de prédicats, quelle que soit la morphologie de ces derniers. Ainsi dans une phrase comme Paul est parti par dépit, on observe deux prédicats du premier ordre : le verbe partir et le prédicat nominal dépit. $\mathrm{Ce}$ que nous venons de dire implique que par dépit ne peut pas être analysé comme un complément de cause mais comme une phrase, dont le prédicat nominal n'est pas actualisé mais qui pourrait l'être grâce au verbe support avoir : Paul est parti parce qu'il avait du dépit. La relation de cause établie entre les deux prédicats du premier ordre est prise en charge par la préposition par, que nous analysons comme un prédicat du second ordre, la structure de base étant que Paul soit parti est par ce qu'il avait du dépit. La soudure de parce que est simplement graphique, comme c'est le cas de afin que / de.

Beaucoup d'autres prépositions doivent être considérées comme des prédicats. Il s'agit au premier plan des prépositions locatives et temporelles. Dans Le livre est sur la table, le prédicat n'est pas le verbe être mais la préposition sur, qui est actualisée par le verbe être ou encore se trouver. De même, Paul est venu avant le départ de Jean repose sur une structure du type Le fait que Paul est venu a eu lieu avant le fait que Jean soit parti (cf. Z. Harris 1976). Un autre exemple est constitué par les prépositions pour et contre dans Paul est (pour, contre) cette décision. Une préposition prédicative a permis ainsi la création d'un verbe : la préposition devant dans: Dans ce classement, Paul est devant Jean a donné naissance au verbe devancer: Dans ce classement, Paul devance Jean. La non prise en compte de ce rôle important des prépositions a entraîné une description inadéquate des subordonnées circonstancielles (G. Gross 1999).

Enfin, certaines prépositions ont une fonction proche de ce que Tesnière appelle une «translation». Elles permettent à partir d'un nom de créer des adjectivaux ou des adverbiaux. Le substantif mode précédé de la préposition à donne l'adjectival à la mode, qui a toutes les propriétés des adjectifs. Il peut être attribut, épithète, apposition; il peut être mis aux différents degrés de comparaison (très à la mode, plus à la mode que) et être anaphorisé par le pronom le tout comme les adjectifs, alors qu'on s'attendrait à la forme en $y$, comme pour les suites introduites par la préposition à. Cette fonction a un intérêt plus étymologique que pratique, puisqu'elle apparaît dans le cas des suites composées qui doivent figurer telles quelles dans un dictionnaire électronique.

\section{Les locutions prépositives introductrices d'arguments}

Nous examinons ici s'il existe des locutions prépositives introduisant des arguments. La difficulté tient à la définition d'un complément d'objet (c'est-à-dire d'un argument) par rapport à un circonstant. Cette question a été maintes fois débattue. Le meilleur critère est le suivant. Un argument ne peut pas être repris par le faire ou par et cela, alors que cette construction est possible pour les circonstanciels : Paul est parti en Amérique (et cela, et il l'a fait) pour gagner de l'argent ; les greniers sont vides (et cela, cela s'est produit) du fait d'une sécheresse inhabituelle. Cette possibilité est exclue pour les arguments des prédicats du premier ordre *Paul a parlé (et cela, il l'a fait) à Jean ; *Paul a discuté avec Jean 
(il l'a fait) de cette histoire de corruption. Ces observations faites, examinons si une locution peut être un indicateur d'arguments. Soit à l'intention de, en faveur de que l'on dit habituellement régir un datif. Or, parallèlement à Paul a dit cela à l'intention de Jean, on peut avoir Paul a dit cela à Luc, à l'intention de Jean. De plus, le critère du circonstanciel signalé plus haut fonctionne tout naturellement Paul a dit cela à Luc et (cela, il l'a fait) à l'intention de Jean. Cette analyse montre que à l'intention de introduit un destinataire plutôt qu'un datif proprement dit. Dans d'autres cas, la situation est plus claire. Avec les prédicats de comportement à l'égard d'autrui, on a le sentiment que certaines locutions introduisent de vrais arguments. Un prédicat comme agressivité semble bien avoir un argument en à l'égard de: Paul a fait preuve d'agressivité à l'égard de Jean, Paul est agressif à l'égard de Jean. D'autres prépositions sont possibles : vis-à-vis de, à l'endroit de.

De même, l'argument-agent en par du passif, dénomination plus judicieuse que celle de " complément d'agent ", peut, dans certaines conditions, se voir substituer une forme complexe : par les soins de, par les bons soins de, par la diligence de: L'affaire a été menée à bien (par, par les soins de, par les bons soins de) l'aîné de la famille. Ces locutions ne font pas partie du sujet proprement dit : *Les bons soins de l'aîné de la famille ont mené à bien cette affaire. On peut en dire autant de locutions comme sous l'effet de : La tôle s'est gondolée sous l'effet du vent ; 'L'effet du vent a fait se gondoler la tôle. On trouvera encore des cas où l'agent est introduit par une locution prépositive : Paul a reçu ce prix (du, des mains du) Président de la République. Dans d'autres cas, on a le sentiment que l'on est en présence de prédicats, comme nous le verrons dans la section suivante : Paul a privilégié Jean au détriment de Luc, qui correspond à Jean a privilégié Paul. Cela s'est fait au détriment de Luc. Les compléments de certains verbes de mouvement sont aussi introduits par des locutions prépositives : se glisser à l'intérieur du tunnel, se retirer à bonne distance de la falaise.

\section{Autres fonctions syntaxiques des locutions prépositives}

Nous montrerons que la notion purement catégorielle de « locution » ne permet pas de mettre en évidence leur diversité syntaxique.

\subsection{Introducteurs locatifs}

Plus de deux cents locutions prépositives ont un sens locatif. Nous analysons ici les compléments dits « circonstanciels ». Les prépositions peuvent être classées selon que l'on a affaire à une localisation interne ou externe. La localisation interne implique que le substantif désigne un élément d'un objet : au dos de, sur le devant de, au revers de, au bout de $\mathrm{N}$, à la face extérieure de $\mathrm{N}$, à la face intérieure de $\mathrm{N}$, à la queue de, à la surface de, à la tête de, à la pointe de, à un bout de $\mathrm{N}$, au bas de $\mathrm{N}$, au beau milieu de, au bord de $\mathrm{N}$, au centre de $N$.

La localisation externe situe un objet par rapport à une scène ou un élément de repère : à deux lieues de là, à deux lieues d'ici, à distance de, à droite de, à l'extrémité de $N$, à l'arrière de $\mathrm{N}$, à l'autre bout de $\mathrm{N}$, à l'écart de $\mathrm{N}$, à l'endroit de N, à l'intersection de et de, à la droite de N, à main droite de, à main gauche de, à mi-hauteur de, à proximité de, au coin de (rue) et (rue), au coin de $N$, au commencement de $N$, au côté de, en arrière de $N$, en aval de, en marge de. 


\subsection{Introducteurs temporels}

pour le lieu, la localisation temporelle se fait par référence au déroulement même d'un événement ou par rapport à un autre événement ou encore à une date. On a alors :

a. la temporalité interne, qui situe un événement dans le déroulement d'un autre : à l'aube de, à la fin de, à l'issue de, au seuil de, en plein milieu de, au plus de, fort de, en plein milieu du réveillon;

b. la temporalité externe qui situe l'événement par rapport à un autre, considéré comme repère. Cet événement peut avoir lieu avant (à la veille de), pendant (du temps de, à l'époque de) et après (à la suite de, au lendemain de) l'événement-repère. Des relations un peu plus complexes peuvent être établies entre les deux événements ;

c. une date : à l'avènement de Louis XIV, au moment de la Révolution.

51 Une étude plus approfondie relèverait plutôt de l'étude des compléments de temps que de celle des locutions prépositives.

\subsection{Introducteurs thématiques}

Il arrive souvent que les propos d'un locuteur dépendent des conditions dans lesquelles il les tient et surtout du point de vue auquel il se place. Celui-ci, pour différentes raisons pragmatiques, prend des précautions oratoires pour replacer son discours dans les conditions requises. Il a à sa disposition un assez grand nombre d'introducteurs thématiques. En voici quelques-uns, traduisant le domaine abordé : dans le domaine de, dans le champ de, au niveau de, en matière de, en fait de, sur le plan de, sur le chapitre de, dans le cadre de, dans le paysage de, dans le contexte de, sur le sujet, sur l'objet, question de, ainsi que le point de vue auquel il se place : du point de vue de, sous l'angle de. Reste le statut théorique de ces unités. Il ne suffit pas de dire que ce sont des introducteurs. Ce terme n'est pas plus précis que celui de « connecteur ", qui n'a pas de statut syntaxique précis. L'hypothèse que nous émettons, c'est que les substantifs qui figurent dans ces locutions sont des variétés de locatifs, arguments de verbes de position : (si on se place, si on se situe, si on se met) (dans le domaine, sur le plan de, au niveau de, dans le cadre de) $N$. Les introducteurs thématiques sont donc un sous-ensemble des constructions locatives. On peut parler à leur propos de lieux abstraits.

\subsection{Réductions de principales d'énonciation}

Un certain nombre de locutions prépositives peuvent être analysées comme des réductions de propositions principales. C'est ainsi qu'il est possible d'analyser De l'avis de Paul, l'affaire est sérieuse où le substantif avis est un prédicat nominal. La phrase signifie approximativement Paul pense que l'affaire est sérieuse. Comme, pour l'analyse, la racine prédicative doit être constante, on aura recours au verbe support être et l'on obtient Paul est d'avis que l'affaire est sérieuse. L'analyse est la même pour de l'aveu de, qui dérive de faire l'aveu que, pour à la demande de qu'on peut tirer de faire la demande, pour sur l'ordre de issu de intimer l'ordre de, pour dans la crainte de obtenu par effacement du verbe être: être dans la crainte de. A l'instigation de est actualisé par être puisqu'il s'agit d'un adjectival : Ce remaniement est à l'instigation de Paul. 


\subsection{Locutions introduisant des subordonnées circonstancielles}

54 prépositive et une locution conjonctive, comme nous l'avons justifié au début de cet article. Les propriétés du substantif fin sont exactement les mêmes dans afin de et afin que, que le substantif soit l'objet de contraintes syntaxiques comme fin ou libre comme dans peur: de peur que, de peur de. Nous allons examiner le statut des substantifs qui figurent dans les locutions introduisant une circonstancielle. Nous les analysons comme des prédicats nominaux d'un type particulier, des prédicats du second ordre. Ils constituent deux classes différentes, selon la nature de leurs arguments phrastiques ou non. Prenons à titre d'exemple les substantifs cause (à cause de) et intention (avec l'intention de).

Partons d'une phrase verbale: Le verglas a causé un terrible accident. Le prédicat peut avoir une forme nominale : Le verglas a été la cause d'un terrible accident. Dans les deux phrases, on peut thématiser l'objet. On obtient alors un passif verbal: Un terrible accident a été causé par le verglas mais aussi nominal : Un terrible accident a été à cause $d u$ verglas. On sait que la préposition à permet de former des passif nominaux : être abrité, être à l'abri de (G. Gross 2001). On voit que la locution à cause de n'a pas de statut propre, qu'il s'agit du prédicat nominal cause dans une configuration syntaxique particulière (passif nominal). Dans tous les cas, le prédicat nominal cause a deux arguments phrastiques, ici deux prédicats événementiels (verglas et accident), comme c'est le cas aussi du verbe causer.

inons maintenant l'autre substantif : Paul est parti en Amérique, avec l'intention de faire fortune, où avec l'intention de est analysé habituellement comme une locution prépositive finale. Il est clair que intention est un prédicat nominal, dont les arguments sont faciles à mettre en évidence. L'objet est la subordonnée infinitive faire fortune et le sujet n'est pas exprimé mais est le même que celui de la principale Paul. De plus, le prédicat intention n'est pas actualisé dans la locution, mais il pourrait recevoir une conjugaison propre grâce au verbe support avoir: Paul est parti en Amérique; il a l'intention de faire fortune. On voit la différence entre les deux prédicats nominaux : cause a deux prédicats phrastiques (événementiels) tandis que intention a un objet phrastique et un sujet humain co-référent à celui de la principale dans la locution.

Cette opposition entre les deux types de constructions ne recoupe pas celle des diverses classes de subordonnées traditionnelles (cause, but, conséquence). Prenons la phrase Paul n'est pas venu sous prétexte qu'il y avait du verglas. Le connecteur sous prétexte que contient le prédicat prétexte qui a le même fonctionnement que intention, bien que la classe sémantique ne soit pas la même. L'objet est une phrase événementielle : il y a du verglas, et le sujet est un humain non exprimé, co-référent à celui du verbe principal. La source est donc Paul n'est pas venu. Il a donné comme prétexte qu'il y avait du verglas.

L'analyse que nous venons d'effectuer s'applique à la plupart des locutions introduisant une subordonnée circonstancielle. On voit donc pourquoi la subordonnée n'est pas facultative : elle représente l'un des arguments du prédicat du second ordre qu'est le substantif prédicatif figurant dans la locution. On renverra sur ce sujet aux analyses que fait Z. Harris (1976), en particulier pour les subordonnées temporelles. 


\subsection{Expression de l'aspect} conjonction $e t$. Il y a beaucoup de moyens lexicaux pour établir cette relation. L'emploi de et implique une égalité entre les deux termes, qui de ce fait, peuvent être permutés : Paul et Jean sont partis au marché; Jean et Paul sont partis au marché. La coordination peut se traduire de même à l'aide de l'adverbe aussi. Dans ce cas, les deux termes ne sont pas sur le même plan. Le second est un ajout au premier, considéré comme prioritaire : Paul est allé au marché; Jean aussi. Ce type de coordination inégale est souvent prise en charge par des locutions prépositives : en compagnie de, en liaison avec, avec l'aide de, en même temps que, conjointement avec, de concert avec.

\section{Conclusion}

Les locutions (prépositives ou conjonctives) ne forment pas une catégorie grammaticale spécifique. On ne peut pas les assimiler à une classe de prépositions composées, au même titre que les noms composés le sont par rapport aux noms simples. Nous avons montré que leur comportement syntaxique est hétérogène. On conclura que le vocabulaire ne peut à lui seul constituer le point de départ de l'analyse linguistique. La syntaxe fait partie de la définition des lexèmes de façon constitutive. Toutes les catégories grammaticales sont syntaxiquement ambiguës. C'est le cas des prépositions 
simples et à plus forte raison des suites appelées «locutions", qui constituent des réalités linguistiques qu'on ne peut pas regrouper en une seule classe. Pour rendre compte de leur diversité, un travail systématique d'analyse s'impose. On verra alors que souvent la dénomination traditionnelle constitue un obstacle à la découverte des réalités linguistiques.

\section{BIBLIOGRAPHIE}

ANSCOMBRE, J.C. (1984), « La représentation de la notion de cause dans la langue », Cahiers de grammaire, 8, pp. 3-53.

CADIOT, P. (1990), « A propos du complément circonstanciel de but », Langue française, 86, pp. 51-64.

DANLOS, L. (1988), « Les expressions figées construites avec le verbe support être Prép », Langages, 90, pp. 23-37.

GINGRAS, L. (1991), « Dans le but de, dans un but de, dans un but (+adj) ", L'actualité terminologique, $24: 1$.

GIRY-SCHNEIDER, J. (1987), Les prédicats nominaux en français. Les phrases simples à verbe support, Genève/Paris, Droz.

GROSS, G. \& PRANDI, M. (éds) (2004) La Finalité. Fondements conceptuels et genèse linguistique, Louvain-la-neuve, De Boeck-Duculot.

GROSS, G. (1988), « Réflexions sur la notion de locution conjonctive », Langue française, 77, pp. 19-36.

GROSS, G. (1989), Les constructions converses du français, Langue et cultures 22, Genève/Paris, Droz.

GROSS, G. (1993), « Trois applications de la notion de verbe support », L'information grammaticale, 59 , pp. 16-22.

GROSS, G. 1994), « Connecteurs et traitement automatique », TA-TAO: Recherches de pointe et applications immédiates, FMA, Beyrouth et AUPELF-UREF (Québec), pp. 287-306.

GROSS, G. (1996a), « Une typologie sémantique des connecteurs : l'exemple de la cause », Studi italiani di linguistica teorica e applicata, XXV :1, Pise, Pacini Editore, pp. 153-179.

GROSS, G. (1996b), « Semantische Umgebung der Konnektoren », Leuvense Bijdragen, 84, pp. 295-311.

GROSS, G. (1999), « Etude lexicale des locutions conjonctives », Mémoires de la Société de Linguistique de Paris, Nouvelle série, T. VII, Peeters, pp. 23-40.

GROSS, G. (2001), « Existe-t-il des verbes supports de type Etre Prép ? », Par monts et par vaux, Mélanges offerts à Martin Riegel, Louvain/Paris Editions Peters, pp. 197-204.

GROSS, G. (2005), « Les circonstancielles sont des complétives », La syntaxe au cœur de la grammaire, recueil offert en hommage pour le $60^{\mathrm{e}}$ anniversaire de Claude Muller, Presses Universitaires de Rennes, pp. 121-127. 
GROSS, G. (1981), « Les bases empiriques de la notion de prédicat sémantique », Langages, 63, pp. 7-52.

GROSS, G. (1986), Grammaire transformationnelle du français - 3. Syntaxe de l'adverbe, Paris, ASSTRILL. GUIMIER, C. (éd) (1993), 1001 circonstants, Caen, Presses Universitaires de Caen.

HARRIS, Z.S. (1976), Notes du cours de syntaxe, Paris, Le Seuil.

LA FAUCI, N. (1988), « Le sujet des conjonctions de subordination finales », Langue française, 77, pp. 37-46.

MEL'CUK, I. (1988), « Principes et critères de description dans le DEC », DEC, 2, Montréal, Les Presses de l'Université de Montréal.

MULLER, C. (1996), La subordination en français, Paris, Armand Colin.

PIOT, M. (1996), Composition transformationnelle des phrases par subordination et coordination, Thèse de l'Université Paris VII.

PRANDI, M. (1994), Le proposizioni finali in italiano : uno studio di grammatica filosofica, manuscrit.

TESNIÈRE, L. (1959), Eléments de syntaxe structurale, Paris, Klincksieck.

VAN KUPPEVELT, J. (1996), « Directionality in Discourse; Prominence Differences in Subordination Relations », Journal of Semantics, 13: 4, pp. 363-395.

VIVÈS, R. (1984), « L'aspect dans les constructions nominales prédicatives : avoir, prendre, verbe support et extension aspectuelle », Lingvisticae Investigationes, VIII : 1, pp. 161-185.

\section{RÉSUMÉS}

Gaston Gross établit les critères définissant la «locution », par opposition à l'expression dite « figée » ou au mot « composé »: son origine (française ou latine), la nature de ses constituants, leurs possibilités de commutation, d'insertion, de variation morphologique, le caractère cataphorique ou anaphorique du déterminant, les diverses fonctions syntaxiques du groupe en question. La conclusion est que « souvent la dénomination traditionnelle constitue un obstacle à la découverte des réalités linguistiques ».

\section{AUTEUR}

\section{GASTON GROSS}

LLI, Université Paris 13 / CNRS 